\title{
Alterstice
}

Revue internationale de la recherche interculturelle

International Journal of Intercultural Research

Revista International de la Investigacion Intercultural

\section{Jumelage interculturel et pédagogie universitaire}

\section{Myra Deraîche, Nicole Carignan et Marie-Cécile Guillot}

Volume 8, numéro 1, 2018

Jumelage interculturel et pédagogie universitaire

URI : https://id.erudit.org/iderudit/1052603ar

DOI : https://doi.org/10.7202/1052603ar

Aller au sommaire du numéro

\section{Éditeur(s)}

Alterstice

ISSN

1923-919X (numérique)

Découvrir la revue

Citer ce document

Deraîche, M., Carignan, N. \& Guillot, M.-C. (2018). Jumelage interculturel et pédagogie universitaire. Alterstice, 8(1), 5-10. https://doi.org/10.7202/1052603ar

\section{Résumé de l'article}

L’année 2001-2002 marque le point de départ de la belle aventure des jumelages interculturels à l'UQAM entre les étudiants francophones de la majorité d'accueil inscrits dans différents programmes et les étudiants immigrants apprenant le français de l'École de langues. À ce jour, c'est plus de 15000 étudiants (jumeaux/jumelles) en travail social, en éducation, en développement de carrière, en communication, en psychologie et en sociologie d'une part et étudiants immigrants apprenant le français d'autre part qui ont bénéficié des jumelages interculturels dans leur formation universitaire. Pour les premiers, cette activité facilite la compréhension des enjeux liés à l'intégration des immigrants, à leur insertion professionnelle et aux rapports intergroupes et interethniques et, pour les seconds, elle facilite l'apprentissage de la langue et de la culture. De plus, les jumelages ont permis la synergie entre une thématique de recherche et une stratégie pédagogique novatrice en enseignement universitaire. Ainsi, le jumelage a le potentiel de contribuer au développement de pratiques pédagogiques où les enseignants et les étudiants apprennent à devenir "interculturellement" compétents. Ce numéro présente un portrait des jumelages interculturels réalisés par des étudiants et leurs professeurs de plusieurs programmes, apprenant ou enseignant différentes langues (français, anglais, espagnol) dans divers contextes universitaires et pays. À l'heure où plusieurs se questionnent sur la capacité d'accueil des immigrants dans leur société respective et des problèmes et risques générés par l'immigration, il est important de proposer des solutions qui montrent qu'il est possible d'accueillir mieux. Le jumelage interculturel est une de ces solutions. 


\title{
7
}

alterstice

Revue Internationale de la Recherche Interculturelle

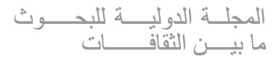

INTRODUCTION THÉMATIQUE

\section{Jumelage interculturel et pédagogie universitaire}

\author{
Myra Deraîche ${ }^{1}$, Nicole Carignan ${ }^{1}$ et Marie-Cécile Guillot ${ }^{1}$
}

\section{Résumé}

L'année 2001-2002 marque le point de départ de la belle aventure des jumelages interculturels à l'UQAM entre les étudiants francophones de la majorité d'accueil inscrits dans différents programmes et les étudiants immigrants apprenant le français de l'École de langues. À ce jour, c'est plus de 15000 étudiants (jumeaux/jumelles) en travail social, en éducation, en développement de carrière, en communication, en psychologie et en sociologie d'une part et étudiants immigrants apprenant le français d'autre part qui ont bénéficié des jumelages interculturels dans leur formation universitaire. Pour les premiers, cette activité facilite la compréhension des enjeux liés à l'intégration des immigrants, à leur insertion professionnelle et aux rapports intergroupes et interethniques et, pour les seconds, elle facilite l'apprentissage de la langue et de la culture. De plus, les jumelages ont permis la synergie entre une thématique de recherche et une stratégie pédagogique novatrice en enseignement universitaire. Ainsi, le jumelage a le potentiel de contribuer au développement de pratiques pédagogiques où les enseignants et les étudiants apprennent à devenir "interculturellement" compétents. Ce numéro présente un portrait des jumelages interculturels réalisés par des étudiants et leurs professeurs de plusieurs programmes, apprenant ou enseignant différentes langues (français, anglais, espagnol) dans divers contextes universitaires et pays. À l'heure où plusieurs se questionnent sur la capacité d'accueil des immigrants dans leur société respective et des problèmes et risques générés par l'immigration, il est important de proposer des solutions qui montrent qu'il est possible d'accueillir mieux. Le jumelage interculturel est une de ces solutions.

\section{Rattachement des auteures}

${ }^{1}$ Université du Québec à Montréal, Montréal, Canada

\section{Correspondance}

deraiche.myra@uqam.ca

\section{Mots clés}

Jumelage interculturel, pédagogie universitaire, formation universitaire, apprentissage d'une langue seconde, éducation interculturelle, compétences de communication interculturelles

\section{Pour citer cet article}

Deraîche, M., Carignan, N. et Guillot, M.-C. (2018). Jumelage interculturel et pédagogie universitaire. Alterstice, 8(1), 5-10. 


\section{Introduction}

Depuis 2002, plus de 15000 jumeaux et jumelles ont participé à des jumelages interculturels organisés à l'Université du Québec à Montréal (UQAM), au Canada. Ces échanges permettent à des étudiants francophones de plusieurs facultés de rencontrer des personnes immigrantes apprenant le français à l'École de langues. Les enseignants impliqués voient depuis longtemps le potentiel de ces activités pour la pédagogie universitaire. D’ailleurs, en 2015, un ouvrage dégageant des assises théoriques pour ces jumelages et proposant la mise en place d'une variété de jumelages a été publié (Carignan, Deraîche et Guillot, 2015). Puis deux colloques internationaux ont mis de l'avant la pertinence, la variété des démarches méthodologiques et les retombées des jumelages interculturels (ACFAS-UQAM, 2016; ACFAS-Sudbury, 2018).

Poursuivant sur cette lancée, ce numéro thématique donne la parole à des chercheurs de l'Australie, du Canada, de la France et du Mexique qui expérimentent des jumelages interculturels dans leurs milieux respectifs et qui en documentent l'apport à la pédagogie universitaire. Avant de présenter leurs articles, il s'avère pertinent de proposer une définition du jumelage interculturel, de dégager le rôle de l'université en matière d'éducation interculturelle et d'explorer les liens entre le jumelage interculturel et la pédagogie universitaire.

\section{Définition du jumelage interculturel}

Le mot jumelage est associé à diverses activités d'échange. En Europe et en Amérique, les jumelages entre villes sont bien connus (Agbobli, 2015). Au Canada, les jumelages interculturels sont pratiqués depuis longtemps dans des organismes communautaires pour l'accueil des personnes immigrantes (Citoyenneté et Immigration Canada, 2010; Guerlotté et Grausem, 2017; Lefebvre-Beaulieu, 2017; Martin, 2002; Picard, 2008; Vatz-Laaroussi et Charbonneau, 2001).

L'activité de jumelage peut être mise en oeuvre dans divers contextes éducatifs. Par exemple, au Québec, l'activité est implantée dans des écoles primaires ou secondaires (Bouchamma, 2015; Petit, 2010), grâce, notamment, au programme d'échanges linguistiques intra-Québec (PÉLIQ-AN) (MEES, 2018) ou dans des établissements du réseau collégial (Coyle, 2017; Gagné et Popica, 2017; Ruel, 2017). La particularité des jumelages interculturels en contexte universitaire est la présence d'un échange entre porteurs de culture dans le cadre d'une formation linguistique (Deraîche, 2014; Guillot, 2017; Zapata et Carignan, 2012) ou professionnelle (Bertrand et Berteau, 2015; Blanchet et Bourhis, 2015; Martiny, 2015).

D'autres substantifs renvoient de près ou de loin au mot jumelage. Ainsi, le mot tandem, qui est souvent employé pour désigner des activités d'échange entre apprenants de langue, met l'accent sur les principes d'autonomie et de réciprocité (Cappellini et Pescheux, 2015). Le jumelage renvoie également à des échanges sur internet, appelés télécollaboration, où des tâches de collaboration entre individus de divers contextes culturels, éducatifs, géographiques et linguistiques sont privilégiées (Derivry-Plard, 2017; O’Dowd, 2018). Ces divers termes montrent que les jumelages se révèlent être des activités souples pouvant se moduler selon les besoins des apprenants, les modalités des programmes de formation et les spécificités des contextes.

En y ajoutant le qualificatif " interculturel ", le jumelage devient le " pairage de personnes de différentes origines ethniques et ou culturelles " (Froelich et Pinard-Prévost, 2014, p. 222), rendant possibles des activités qui valorisent la reconnaissance mutuelle, motivent la communication et brisent l'isolement de certains individus.

\section{Rôle de l'université en matière d'éducation interculturelle}

L'université peut jouer un rôle en donnant son appui aux membres de sa communauté pour établir le contact et le dialogue interculturel. Ainsi, pour Wächter (2009), l'université devrait assumer deux rôles pour engendrer et soutenir la compréhension interculturelle : 1) favoriser le dialogue sur les campus en y inculquant « une culture institutionnelle de la sensibilité interculturelle » (p. 150) et 2) susciter l'échange dans la société en général. D'autre part, Lehtomäki, Moate et Posti-Ahokas (2016) pensent que la tâche des institutions universitaires est de préparer les futurs professionnels à comprendre finement la valeur ajoutée de la diversité. De plus, ces chercheurs affirment que les cultures disciplinaires et les pratiques pédagogiques arrivent difficilement à créer des situations d'apprentissage 
favorisant le dialogue interculturel. Pour eux, il devient crucial de créer des interventions ciblées, planifiées et structurées, en contexte universitaire et avec le soutien des établissements, pour bâtir des relations interculturelles positives sur les campus.

Les expériences de jumelage interculturel proposées ici avivent ce débat sur le dialogue interculturel et l'inclusion de tous les étudiants universitaires. Ces jumelages permettent également de s'interroger sur le rôle des institutions, des départements, des services et de divers acteurs de la communauté universitaire.

\section{Jumelage interculturel et pédagogie universitaire}

Les jumelages interculturels s'avèrent une pratique pédagogique innovante. Si l'on en croit Lison, Bédard, Beaucher et Trudelle (2014), tout ce qui ne relève pas de l'enseignement magistral peut être considéré comme novateur en pédagogie de l'enseignement supérieur. En effet, même si on sait depuis longtemps que le rôle des professeurs " n'est plus de transmettre des connaissances, mais de soutenir la compréhension, la construction et l'intégration des savoirs par leurs étudiants " (Lison et Jutras, 2014, p. 3), rares sont ceux qui s'écartent de la présentation orale des contenus en contexte universitaire. Toute pratique pédagogique qui sort de cette norme, comme le jumelage interculturel, peut ainsi être qualifiée d'innovation.

En matière de pratiques pédagogiques novatrices, Berteau et Martiny (2015) soulignent l'apport de la pédagogie humaniste pour les jumelages interculturels entre des étudiants en travail social et en counseling. Les échanges interculturels à l'université sont aussi associés à une pédagogie qui encourage « le développement de la décentration, dans une perspective interculturelle, chez les futurs enseignants " (Steinbach, 2016). Aussi, Bourhis, Carignan et Sioufi (2015) rappellent que les stratégies pédagogiques qui ont un impact positif reposent sur la complémentarité des aspects émotionnels et cognitifs des modes de communication. En effet, lors des jumelages, les aspects émotionnels sont activés par tout ce que les étudiants expérimentent, comme les témoignages relatant des situations d'injustice ou d'inégalité ou de discrimination, tandis que les aspects cognitifs relèvent de tout ce que les étudiants partagent comme informations factuelles qu'ils apprennent les uns des autres. Enfin, Abdallah-Pretceille (2010) met de l'avant la pédagogie interculturelle, qui est une pédagogie axée sur l'échange " entre » individus de différentes cultures et non " sur ces » individus. Les jumelages suivent ainsi la voie de la pédagogie interculturelle centrée sur la relation, la communication avec l'Autre, au lieu de favoriser les descriptions et les comparaisons culturelles.

\section{Présentation des articles}

Dans le premier article, Alhassane Balde aborde la question de la pédagogie de l'ouverture à l'autre. II met en lumière l'expérience d'étudiants futurs enseignants d'une université canadienne qui ont suivi deux cours centrés sur la pédagogie donnés par le même enseignant : gestion de classe et éducation interculturelle. L'étude fait ressortir que l'enseignant a pu constamment établir des liens entre les deux cours et que les activités de jumelage ont consolidé les acquis de ces deux cours. À titre d'exemple, les étudiants, sensibilisés aux situations de discrimination, ont été encouragés à concevoir des activités assurant l'accompagnement de " tous » leurs élèves dans une approche inclusive.

Dans la deuxième recherche, Marie-Cécile Guillot et Nicole Carignan rappellent le peu de contact en contexte québécois des immigrants avec la communauté francophone majoritaire. Cette situation constitue un frein important à leur apprentissage et à leur usage du français. L'article présente un jumelage au Canada entre des étudiants qui suivent un cours en rédaction du français et de futurs enseignants suivant un cours d'éducation interculturelle. Les auteures y rappellent l'importance de susciter un climat de confiance mutuel entre enseignants, entre enseignants et étudiants et entre étudiants.

Dans le troisième texte, Valérie Amireault et Soline Trottet sortent des pratiques habituelles de jumelage en contexte de formation à l'enseignement. Dans leur cas, la rencontre interculturelle s'effectue par l'exploitation de matériel littéraire. Ainsi, des étudiants universitaires au Mexique participent à un projet interculturel de théâtralisation d'une légende québécoise. Les diverses activités du projet se révèlent créer un espace de découverte et de réflexion sur la culture québécoise pour ces étudiants en enseignement du français langue étrangère (FLE). 
Avec l'étude de Jessica Payeras et de Maria Elena Zapata, le numéro thématique sort encore une fois des sentiers battus. Dans cet article rédigé en espagnol (avec une synthèse en français), les enseignantes de l'espagnol langue étrangère (ELE) font part de leur projet d'échanges linguistiques et interculturels qui ont lieu au Canada. Ces activités réunissent des francophones apprenant l'espagnol à l'université et des hispanophones nouvellement arrivés au Québec inscrits à un programme de francisation. Ces jumelages poursuivent, entre autres, l'objectif de dissiper les préjugés interculturels développés lors de l'apprentissage des deux langues, l'espagnol et le français.

L'avant-dernier texte porte sur des interactions en tandem anglais/français dans un contexte européen. II s'agit d'activités d'échange entre des étudiants francophones d'une université française et des participants anglophones en séjour en France. Les auteures, Céline Horgues et Sylwia Sheuer, procèdent à l'analyse des interactions pour en examiner plus précisément les rétroactions correctives. Cette expérimentation fait ressortir, notamment, l'influence des traditions éducatives et culturelles.

Dans le dernier article, celui de Myra Deraîche et de Christèle Maizonniaux, les jumelages interculturels prennent le virage des nouvelles technologies. II s'agit d'un projet de télécollaboration entre des apprenants de français langue étrangère (FLE) en Australie et des personnes immigrantes apprenant le français langue seconde (FLS) au Canada. Les étudiants universitaires expérimentent des échanges interculturels riches sur internet en exploitant des textes de la littérature migrante francophone et en explorant les thèmes de l'exil et de la biographie.

En somme, ce numéro présente un portrait des jumelages interculturels pratiqués dans différents pays et contextes universitaires. L'équipe éditoriale vous souhaite une agréable et enrichissante lecture de ces projets pédagogiques novateurs.

\section{Remerciements}

Nous remercions la Faculté des sciences de l'éducation de l'Université du Québec à Montréal (UQAM). Pour la publication de ce numéro thématique, une subvention a été obtenue par Myra Deraîche dans le cadre du Programme d'aide à la recherche pour les étudiants de la Faculté des sciences de l'éducation.

\section{Références bibliographiques}

Abdallah-Pretceille, M. (2010). L'éducation interculturelle ( $3^{\mathrm{e}}$ éd.). Paris : Presses Universitaires de France.

ACFAS-UQAM (2016). Rencontres interculturelles comme pistes de développement de la pédagogie postsecondaire : perspectives internationales. Montréal (Québec, Canada), 10 et 11 mai 2016.

ACFAS-Sudbury (2018). Les jumelages interculturels en partage \Sharing intercultural twinnings. Sudbury (Ontario, Canada), 6 avril 2018,.

Agbobli, C. (2015). Postface. Dans N. Carignan, M. Deraîche et M.-C. Guillot (dir.), Jumelages interculturels : communication, inclusion et intégration (p. 145-147). Montréal : Presses de l’Université du Québec.

Berteau, G. et Martiny, C. (2015). Contact intergroupe et aide mutuelle dans le jumelage en travail social, carriérologie et français langue seconde. Dans N. Carignan, M. Deraîche et M.-C. Guillot (dir.), Jumelages interculturels : communication, inclusion et intégration (p. 53-62). Montréal : Presses de l'Université du Québec.

Bertrand, J. et Berteau, G. (2015). Jumelage pour la communication orale et le travail de groupe. Dans N. Carignan, M. Deraîche et M.-C. Guillot (dir.), Jumelages interculturels : communication, inclusion et intégration (p. 91103). Montréal : Presses de l'Université du Québec.

Blanchet, J. et Bourhis, R. (2015). Jumelage par entrevue dans un cours de phonétique et un cours en psychologie. Dans N. Carignan, M. Deraîche et M.-C. Guillot (dir.), Jumelages interculturels : communication, inclusion et intégration (p. 75-89). Montréal : Presses de l'Université du Québec.

Bouchamma, Y. (2015). L'école et l'immigration. Défis et pratiques gagnantes. Lévis : Les Éditions de la Francophonie. 
Bourhis, R., Carignan, N. et Sioufi R. (2015). Acculturation et jumelage interculturel dans la formation à l'enseignement. Dans N. Carignan, M. Deraîche et M.-C. Guillot (dir.), Jumelages interculturels : communication, inclusion et intégration (p. 15-32). Montréal : Presses de l'Université du Québec.

Cappellini, M. et Pescheux, M. (2015). La gestion des normes langagières dans un tandem franco-chinois par visioconférence. Bulletin VALS-ASLS, 3, 171-187.

Carignan, N., Deraîche, M. et Guillot, M.-C. (2015). Jumelages interculturels : Communication, inclusion et intégration. Montréal : Presses de l'Université du Québec.

Citoyenneté et Immigration Canada. (2010). Évaluation du Programme d'accueil. Ottawa : Citoyenneté et Immigration Canada.

Coyle, S. (2017). Virtual Team Teaching. A Collaborative Approach. Montréal : Chenelière Éducation.

Deraîche, M. (2014). Les jumelages interculturels. Portrait d'une pratique avec des immigrants en classe de FLS. La Revue de l'AQEFLS, 31(1), 93-106.

Derivry-Plard, M. (2017). Introduction. Les Langues Modernes, (janvier-février-mars), 8-13.

Froelich, A. et Pinard-Prévost, G. (2014). La formation à l'éducation interculturelle des enseignants au Québec : de la tolérance à la reconnaissance. Compte-rendu d'une expérience d'échange interculturel. Dans D. Alaoui et A. Lenoir (dir.), L'interculturel et la construction d'une culture de la reconnaissance (p. 213-234). Longueuil : Groupéditions.

Gagné, P. et Popica, M. (2017). Perceptions et motivation à l'égard du français langue seconde enseigné au Québec [en ligne]. Montréal : EDUQ.info. https://eduq.info/xmlui/handle/11515/35311

Guerlotté, C. et Grausem, D. (2017). Le jumelage interculturel au Québec. Montréal : Table de concertation des organismes au service des personnes réfugiées et immigrantes.

Guillot, M.-C. (2017). Les jumelages interculturels dans un cours de français langue seconde. Le français dans le monde, 409, 36-37.

Lefebvre-Beaulieu, L. (2017). Les pratiques de jumelage interculturel en contexte pluriethnique québécois : Comment renforcer le lien social au regard de la médiation interculturelle. Sherbrooke : Université de Sherbrooke.

Lehtomäki, E., Moate, J. et Posti-Ahokas, H. (2016). Global connectedness in higher education: student voices on the value of cross-cultural learning dialogue. Studies in Higher Education, 41(11), 2011-2027. https://doi.org/10.1080/03075079.2015.1007943

Lison, C., Bédard, D., Beaucher, C. et Trudelle, D. (2014). De l'innovation à un modèle de dynamique innovationnelle en enseignement supérieur. Revue Internationale de Pédagogie de l'enseignement Supérieur, 30(1), 1-20.

Lison, C. et Jutras, F. (2014). Innover à l'université : penser les situations d'enseignement pour soutenir l'apprentissage. Revue Internationale de Pédagogie de l'Enseignement Supérieur [en ligne], 30(1). https://journals.openedition.org/ripes/769

Martin, A. (2002). Le jumelage entre les nouveaux arrivants et les Québécois de la société d'accueil. Québec : Université Laval. http://hdl.handle.net/20.500.11794/17780

Martiny, C. (2015). Approche orientante et jumelage en carriérologie. Dans N. Carignan, M. Deraîche et M.-C. Guillot (Eds.), Jumelages interculturels : communication, inclusion et intégration (p. 63-72). Montréal : Presses de l'Université du Québec.

Ministère de l'Éducation et de l'Enseignement supérieur du Québec (MEES). (2018). Programme d'échanges linguistiques intra-Québec (PÉLIQ-AN). Approche nouvelle [en ligne]. Consulté le 13 juin 2018. http://blogdev.learnquebec.ca/peliqan/fr/a-propos-de-peliq-an/ 
O'Dowd, R. (2018). From telecollaboration to virtual exchange: state-of-the-art and the role of UNICollaboration in moving forward. Journal of Virtual Exchange, 1(0), 1-23.

Petit, V. (2010). Les jumelages linguistiques et culturels entre écoles francophones et anglophones : qu'en pensent les parents? (thèse de doctorat, Université de Sherbrooke, Sherbrooke). https://savoirs.usherbrooke.ca/handle/11143/2820

Picard, Y. (2008). Faux jumeaux, vrais amis. L'Actualité, 33(19), 76-78.

Ruel, H. (2017). La grande leçon des jumelages interculturels. La Nouvelle union, [15 février]. https://www.lanouvelle.net/la-grande-lecon-des-jumelages-interculturels/

Steinbach, M. (2016). Les échanges interculturels pour promouvoir la compétence interculturelle. Dans M. Potvin, M.-O. Magnan et J. Larochelle-Audet (dir.), La diversité ethnoculturelle, religieuse et linguistique en éducation (p. 134-136). Montréal : Fides.

Vatz-Laaroussi, M. et Charbonneau, J. (2001). L'accueil et l'intégration des immigrants : à qui la responsabilité? Le cas des jumelages entre familles québécoises et familles immigrantes. Lien Social et Politiques, 46, 111-124. https://doi.org/10.7202/000327ar

Wächter, B. (2009). Le dialogue interculturel sur le campus universitaire. Dans Dialogue interculturel sur les campus universitaires (p. 145-152). Strasbourg: Éditions du Conseil de l'Europe.

Zapata, M. E. et Carignan, N. (2012). Les jumelages linguistiques : une expérience d'interculturalité à Montréal. Canadian Diversity/Diversité Canadienne, 9(2), 52-56. 